\title{
CEFUROXIME-INDUCED THROMBOCYTOPENIA: IT'S JUST NOT IN THE RING??
}

\author{
BALAJI O, NAVIN PATIL*, AVINASH A, AMOD TILAK
}

Department of Pharmacology, KMC, Manipal, Karnataka, India. Email: navin903@gmail.com

Received: 27 May 2016, Revised and Accepted: 02 June 2016

\begin{abstract}
Beta lactams are one of the most commonly used antibiotic groups in clinical practice, owing to their relatively superior safety profile, when weighed against other available antibiotics. Cephalosporins have overgrown over the years, now extending across five generations of drugs. Older cephalosporins are still commonly used, chiefly because of their low cost and minimal adverse effects. However, no drug is entirely free of adverse effects. Cephalosporins (especially those with a methylthiotetrazole ring) have been associated with bleeding manifestations due to hypoprothrombinemia and thrombocytopenia in susceptible subsets of the global population. This case report concerns the use of a cephalosporin that does not have this ring in its structure but has still caused a rare instance of thrombocytopenia.
\end{abstract}

Keywords: Beta lactam, Cefuroxime, Platelets, Bleeding, Methylthiotetrazole.

(C) 2016 The Authors. Published by Innovare Academic Sciences Pvt Ltd. This is an open access article under the CC BY license (http://creativecommons. org/licenses/by/4. 0/) DOI: http://dx.doi.org/10.22159/ajpcr.2016.v9i5.13112

\section{INTRODUCTION}

Drug-induced thrombocytopenia (DIT) is a very common adverse reaction, and hence, it is very important to identify and remove the offending agent to avoid life-threatening episodes of bleeding or thrombosis. DIT can be mainly due to two reasons: Either immunemediated (increased destruction of platelets) or due to suppression of bone marrow (reduced formation of platelets) [1]. History of drug intake and laboratory investigations usually helps in distinguishing DIT from Immune Thrombocytopenic Purpura (ITP) [2]. Among the commonly used beta-lactam antibiotics, cephalosporins with a thiomethyltetrazole ring in their chemical structure have been known to be associated with hypoprothrombinemia and thrombocytopenia. The authors report a case of cefuroxime-induced thrombocytopenia, a $2^{\text {nd }}$ generation cephalosporin that does not possess the thiomethyltetrazole ring structure. An extensive search of the available literature revealed only one such report till date [3].

\section{CASE REPORT}

A 57 years old alcoholic male patient (informed consent obtained) presented with complaints of difficulty in walking, slurred speech, hallucinations, swaying to both sides while walking, with bilateral occipital spike discharge. He was diagnosed of alcohol withdrawal syndrome and was started on oral chlordiazepoxide and olanzapine, both administered once daily.

After 20 days, the patient was diagnosed of drug-induced extrapyramidal symptoms (EPS), and hence, olanzapine was discontinued. He was started instead on oral quetiapine once daily. Following this, the patient exhibited a gradual recovery from the symptoms and signs of EPS. However, he developed cough, fever, respiratory distress, and breathlessness, raising the suspicion of hospital acquired infection, owing to his prolonged stay in the hospital. Polymerase chain reaction was done to rule tuberculosis, and sputum culture was negative for acidfast bacilli. Another sputum culture was performed, which confirmed methicillin-sensitive Staphylococcus aureus. Urine culture revealed the growth of Pseudomonas spp. All the other blood parameters were normal.

Based on a drug sensitivity testing, the patient was started on intravenous (IV) cefuroxime at a dose of $750 \mathrm{mg} 8^{\text {th }}$ hourly. 3 days later, it was noted that the patient's platelet count was low when compared to his initial platelet counts before the start of treatment (Table 1). The patient was not on any other concomitant medications that could have caused a drop in platelet count. Other tests were done to rule out dengue, malaria, and hemorrhagic fever that can cause thrombocytopenia. All tests were negative, and hence, cefuroxime-induced thrombocytopenia was suspected. Cefuroxime was discontinued immediately, and the patient was started with IV meropenem $1 \mathrm{~g}$ IV $8^{\text {th }}$ hourly for 7 days. No specific treatment was initiated for tAQAQhrombocytopenia. The patient's condition recovered after a fortnight of stopping cefuroxime as evident from Table 1.

\section{DISCUSSION}

Thrombocytopenia is defined as a platelet count of $<150 \times 10^{3} / \mu \mathrm{L}$. The extent of the drop in the platelet count determines the symptoms per se, and hence the type of clinical presentation. With a count of $<30 \times 10^{3} / \mu \mathrm{L}$, bleeding symptoms are common and may manifest as purpura, gum bleeds, hematuria, hematemesis, etc., Very low counts may lead to lifethreatening bleeding complications. The etiology of thrombocytopenia is varied and usually requires further blood investigations. Common causes include acute infections, bone marrow failure, liver disease, autoimmune disorders, thrombotic thrombocytopenic purpura, hemolytic uremic syndrome, disseminated intravascular coagulation, and drugs such as heparin. DIT is suspected in patients with isolated thrombocytopenia and should be differentiated from ITP and pseudothrombocytopenia. Treatment of the condition is broadly based on the etiology, and in some cases, treatment of the underlying cause is necessary [4].

DIT has been reported to have an annual incidence of 10 cases per 1 million population [5]. If DIT is suspected, the potential causative drug should be identified and discontinued immediately. Improvement usually occurs within 2 weeks of discontinuation. Common drugs that have been implicated in DIT include heparin, carbamazepine, cephalosporins (which contain the thiomethyltetrazole ring), vancomycin, abciximab, cimetidine, hydrochlorothiazide, interferons, phenytoin, quinidine, quinine, rifampin, co-trimoxazole, among several others [6]. Pathogenetic mechanisms involved in DIT are chiefly immunological: Hapten-induced antibodies, autoantibodies, drug-dependent antibodies, drug-specific antibodies, ligand-mimetic processes, and immune complex formations [7]. Diagnosis of DIT is usually empirical, and improvement of platelet count after discontinuation (dechallenge) of offending agent is often taken as circumstantial evidence. Platelet immunofluorescence test, enzyme-linked immunospecific assay, flow cytometry, and immunoprecipitation-Western blotting are some of the laboratory tests employed to diagnose DIT [1]. 
Table 1: Platelet count before, after starting, and stopping the drug

\begin{tabular}{ll}
\hline Day/timing & Platelet count \\
\hline Day 0 (at the start of drug) & $303 \times 10^{3} / \mu \mathrm{L}$ \\
Day 3 (drug stopped) & $122 \times 10^{3} / \mu \mathrm{L}$ \\
Day 4 (after stopping drug) & $102 \times 10^{3} / \mu \mathrm{L}$ \\
Day 15 (after stopping drug) & $372 \times 10^{3} / \mu \mathrm{L}$ \\
\hline
\end{tabular}

Table 2: Adverse drug reaction assessment and scaling

\begin{tabular}{lll}
\hline Naranjo's scale & Hartwig's scale & Thornton's scale \\
\hline Probable causality & Moderately severity & Not preventable \\
\hline
\end{tabular}

Cephalosporins are beta-lactam antibiotics that interfere with bacterial cell wall synthesis. Changes in the R2 side chain of these compounds bring about significant pharmacokinetic disposition and toxicity. $\mathrm{N}$-methylthiotetrazole moiety is found in a few cephalosporins such as cefamandole, cefoperazone, and cefotetan. Drugs with this side chain have been commonly associated with hypoprothrombinemia and disulfiram-like reactions. The same is also substantiated as the cause for thrombocytopenia found among these $3^{\text {rd }}$ generation cephalosporins [8].

In the current case, the cephalosporin that caused thrombocytopenia was cefuroxime, which is a $2^{\text {nd }}$ generation cephalosporin (absence of $\mathrm{N}$-methylthiotetrazole moiety). Adverse effects associated with the use of cefuroxime include hypersensitivity reactions, pain at the site of injection, gastrointestinal disturbances, pseudomembranous colitis, anemia, leucopenia, transient derangement of hepatic markers. In addition, we could locate one report of thrombocytopenia following the use of cefuroxime. The mechanism by which cefuroxime could cause thrombocytopenia might be hypothesized to be immunological in nature: Probably due to an increase in drug-dependent plateletassociated IgG antibodies [2]. In our case, the patient's condition improved, with platelet count returning to normal within a fortnight after discontinuing the drug, thus confirming to a certain extent that the drug in question was the culprit. Causality assessment was done as per
Naranjo's scaling (Table 2), and a "probable" causal relationship was established [9]. The adverse drug reaction was found to be "moderately severe" and "not preventable" (Table 2) as per Hartwig's severity scaling and Thornton's preventability scaling, respectively $[10,11]$.

\section{CONCLUSION}

Based on the literature review conducted, DIT with $2^{\text {nd }}$ generation cephalosporins is extremely rare. When isolated thrombocytopenia occurs in patients on cefuroxime, all common causes should be ruled out to confirm the diagnosis of DIT. The drug should be discontinued immediately and never be rechallenged because life-threatening bleeding complications may occur. In the future, cohort studies of various cephalosporins causing thrombocytopenia can be undertaken to have a better knowledge of this adverse drug reaction in different populations.

\section{REFERENCES}

1. Visentin GP, Liu CY. Drug-induced thrombocytopenia. Hematol Onco Clin North Am 2007;21(4):685-96.

2. Pathak A, Kunder SK, Avinash A, Patil N, Rao NK. A rare case of tamoxifen-induced thrombocytopenia. J App Pharm Sci 2016;6(1):156-7.

3. Aitken P, Zaidi SM. Cefuroxime-induced thrombocytopenia? Postgrad Med J 1996;72(854):757-8

4. Gauer RL, Braun MM. Thrombocytopenia. Am Fam Physician 2012;85(6):612-22.

5. van den Bemt PM, Meyboom RH, Egberts AC. Drug-induced immune thrombocytopenia. Drug Saf 2004;27(15):1243-52.

6. Kenney B, Stack G. Drug-induced thrombocytopenia. Arch Pathol Lab Med 2009;133(2):309-14

7. Aster RH. Drug-induced immune cytopenias. Toxicology 2005;209(2):149-53.

8. Kalman D, Barriere SL. Review of the pharmacology, pharmacokinetics, and clinical use of cephalosporins. Tex Heart Inst J 1990;17(3):203-15.

9. Naranjo CA, Busto U, Sellers EM, Sandor P, Ruiz I, Roberts EA, et al. A method for estimating the probability of adverse drug reactions. Clin Pharmacol Ther 1981;30(2):239-45.

10. Hartwig SC, Siegel J, Schneider PJ. Preventability and severity assessment in reporting adverse drug reactions. Am J Hosp Pharm 1992;49(9):2229-32.

11. Schumock GT, Thornton JP. Focusing on the preventability of adverse drug reactions. Hosp Pharm 1992;27(6):538. 